\title{
Saudi Arabia Experience in Implementing Telemental Health during COVID-19 Pandemic
}

\author{
Weam M. Banjar ${ }^{\mathrm{a}}$ Amjad Alfaleh ${ }^{\mathrm{b}, \mathrm{c}}$ \\ ${ }^{a}$ Global Center for Mass Gathering Center, Ministry of Health, Riyadh, Saudi Arabia; ${ }^{b}$ General Directorate for School \\ Health, Ministry of Health, Riyadh, Saudi Arabia; 'General Directorate for Medical Consultation, Ministry of Health, \\ Riyadh, Saudi Arabia
}

As of March 24, 2021, the total number of confirmed coronavirus disease 2019 (COVID-19) cases reached 386,300 cases in Saudi Arabia with a 97.20\% recovery rate and a $1.71 \%$ mortality rate. Globally, the number of confirmed cases exceeded 100,000,000 cases with a 56.73\% recovery rate and a $2.20 \%$ mortality rate [1]. The first COVID-19 case was reported in December 2019 in Wuhan, China [2,3]. The WHO declared COVID-19 outbreak a public health emergency with international concern in January 30,2020, in response to global increase of COVID-19 cases [4]. In March 11, 2020, the WHO declared COVID-19 pandemic [5]. In order to contain the pandemic, health authorities recommended physical social distancing and limiting community movement [6]. While these are steps necessary to control the infection spread, population will face mental and well-being consequences in short and long terms. In the absence of medical cure for COVID-19, the global response adapted a number of public health protection measures that focus on isolation of suspected and confirmed cases, reduced social contact, vaccination, and simple hygiene practices [7]. Containment strategies together with rapid changing evidence about COVID-19 may affect the population psychological well-being. Coping with illness of self, fam-

karger@karger.com www.karger.com/sjh

Karger"
(C) 2021 The Author(s)

Published by S. Karger AG, Basel

This is an Open Access article licensed under the Creative Commons Attribution-NonCommercial-4.0 International License (CC BY-NC) (http://www.karger.com/Services/OpenAccessLicense), applicable to the online version of the article only. Usage and distribution for commercial purposes requires written permission. ily, or significant one during home isolation or hospitalization may be stress-provoking, and with uncertainty linked to the course of the pandemic, it is expected that mental health burden will worsen over time [6]. COVID-19 patients reported elevated stress levels due to limited personal interaction, fear, and social and financial stresses $[6,8]$. The psychological impact of COVID-19 must be recognized alongside the surveillance for physical symptoms and implementation of public health protection measures $[7,9]$. The pressure on healthcare workers (HCWs) continued to increase alongside with perceived lack of influence on decision-making which rises tension $[10,11]$. Frontline HCWs face multiple challenges that include unexpected workload, rising occupational hazard, and social stresses. Frontline HCWs' psychological response to an increased work tension is pivotal to maintain the healthcare system operational efficiency.

Mental health care is important during the pandemic. The COVID-19 pandemic may worsen existing mental health conditions, and disruption of care may exacerbate symptoms. COVID-19 mental health impact is linked to the unique combination of mental health services, physical social distancing, and economic recession [12]. The increasing demand on mental health services in the pe-
Correspondence to:

Weam M. Banjar, wbanjarm@moh.gov.sa 
riod of overwhelmed mental healthcare system presents a risk for creating a mental health crisis globally $[13,14]$. Thus, differentiation between emergency mental care and routine mental care is essential to design an appropriate model that establishes effective care delivery mode and defines operational scheme.

There is an urgent need for establishing a feasible platform that integrates technologies and innovative mental healthcare model. Digital health technologies supported healthcare services delivery at the time of crisis. Telemedicine is defined as the delivery of care and services, where distance is a critical factor, using information and communication technologies [13, 15]. When telemedicine principles are applied to psychiatric/mental health care, it is termed telepsychiatry or telemental health. Telemental health refers to the utilization of information and communication technologies to facilitate delivery of mental healthcare remotely for evaluation, medication management, and psychotherapy $[6,16]$. Telemental health could be the ideal solution to reduce the risk of infection while maintaining continuity of care. Telemental health can respond to mental health needs of people in isolation, quarantine, and/or during restricted mobility.

Treatment protocols for COVID-19 patients should address both physiological and psychological needs. Telemental health services will help patients maintain psychological well-being and cope with acute and postacute health requirement [3]. Availability of and accessibility to psychological care and support programs during the pandemic may reduce the burden of comorbid mental health conditions and ensure population well-being [3]. Telemental health services are suitable for the pandemic situation because it gives people in remote location access to care while reducing the risk of cross-infection [3]. Research on telephone-delivered psychotherapy had been found as effective as face-to-face therapy session $[12,17$, 18]. Telemental health services can be delivered via video conferencing, smartphone apps, online forums, and/or e-mails.

The rise in the number of COVID-19 cases is accompanied by increased fear and pressure among HCWs and general population. Thus, mental health care during the pandemic is essential. The Ministry of Health $(\mathrm{MOH})$ in Saudi Arabia acknowledged the impact of the pandemic on the population mental health and quickly realized the need to establish a platform that meets the increasing demands to ensure the psychological well-being of the general population and the continuity of care and offers a secured government-sponsored channel to deliver mental health care. The public health protection strategies were implemented to mitigate the risk of cross-infection, and the center of telemedicine at $\mathrm{MOH}$ supported the "With You" (MAKOM) initiative. The initiative aims to deliver telemental health services through a secured platform that preserves end-users confidentiality.

A total of 128 mental health professionals were recruited to volunteer to deliver telemental health services. The recruitment process involved evaluation of candidates experience and qualification. Selected candidates received intensive training to ensure quality of services intended to deliver. Between the period of April 22, 2020, and March 6, 2021, a total of 38,650 psychological consultations had been delivered. Anxiety, depression, stresses due to COVID-19, sleep disorders, and panic attacks were the most common mental health disorders presented to telemental health consultation services. In order to ensure delivery of proper service, callers went through a triage process prior to referral to telemental health consultation. The services were delivered 24/7.

Telemental health services had been launched in response to meet the need to combat psychological impact of COVID-19 and to avoid disruption of care as a result of preventive strategies. Insufficient infrastructure in terms of human resources and technological support prevented from benefiting from all telemental health service features. Due to limited technical support, the consultation time was kept to minimal to avoid any service disruption. Patients' information and data confidentiality and privacy presented another challenge. Due to lack of integration between telemental health services and patients' medical records, assessment of patients' mental health and medication history was not possible, and therefore medication management and prescriptions became a challenge. Limited consultation time, lack of access to patients' medical records, and social dilemma of psychological disorders together presented a challenge to mental health professionals to arrive at proper diagnosis. In order to maintain service quality and sustainability, it is pivotal to establish the governance guidelines that ensure monitoring and continuous assessment of adherence to scope to services and clinical protocols.

Launching telemental health services during COVID-19 pandemic was an enriching experience that led to advance the movement to expand the implementation of telemental health services to complement routine faceto-face services. Regardless of initial reluctance to utilize the services, positive feedback had been reported by patients. Telemental health is essential in time of crisis; however, it is pivotal to consider implementation of services alongside office-based mental health care. Telemen- 
tal health service ensures a safe interactive professional environment that maintains continuity of care, reduces the cost, and improves quality of life.

The Saudi telemental health experience during COVID-19 pandemic reflects the need to establish the infrastructure landscape from technical, legislative, and human resources aspects. Policy makers, mental health professionals, and public health professionals should support the framework for the provision of telemental health services during crisis and beyond. The role of mental health professional societies and nongovernmental organizations should be acknowledged to define the service scope and expand the impact horizon. Mental health training programs need to equip trainees with skills and quality to deliver telemental health services alongside routine services. Telemental health in Saudi Arabia is at its infancy. However, holistic approach should be considered to support initiatives that aim at adoption of telemental services to improve quality of care and service accessibility with consideration of ethical and confidentiality requirement. Access to mental health is essential for population wellbeing. However, telemental health experience during
COVID-19 sets the foundation for establishing comprehensive telemental health services with immediate access to interventional and therapeutic for those seeking assistance especially when accessibility is an issue particularly in remote areas and among health disparities. Telemental health services complement routine clinical visits; however, ethical and confidentiality challenges must be carefully assessed and addressed to ensure efficacy of services.

\section{Conflict of Interest Statement}

There are no conflicts of interest to declare.

\section{Funding Sources}

No funding had been received for this project.

\section{Author Contributions}

Both authors contributed equally to the manuscript in writing, reviewing contents, and approving the manuscript.

\section{References}

1 Public Health Authority (COVID-19) Disease Interactive Dashboard. Daily updates [cited 2021 Mar 25]. Available from: https:// covid19.cdc.gov.sa/daily-updates/.

2 Sharma A, Sasser T, Schoenfelder Gonzalez E, Vander Stoep A, Myers K. Implementation of home-based telemental health in a large child psychiatry department during the COVID-19 crisis. J Child Adolesc Psychopharmacol. 2020;30(7):404-13

3 Zhu N, Zhang D, Wang W, Li X, Yang B, Song $\mathrm{J}$, et al. China novel coronavirus investigation and research team: a novel coronavirus from patients with pneumonia in China, 2019. N Engl J Med. 20201;382:727-33.

4 World Health Organization (WHO). Novel coronavirus (2019-nCOV) situation report-10. 2020 [cited 2021 Mar 25]. Available from: https://www.who.int/docs/defaultsource/coronaviruse/situationreports/20200130-sitrep-10-ncov.pdf? sfvrsn $=$ d0b2e480 2 .

5 World Health Organization (WHO). Coronavirus disease 2019 (COVID-19) situation report-51. 2020 [cited 2021 Mar 25]. Available from: https://www.who.int/docs/default-source/coronaviruse/situationreports/20200311-sitrep-51-covid-19. pdf?sfvrsn=1ba62e57_10.
6 Whaibeh E, Mahmoud H, Naal H. Telemental health in the context of a pandemic: the COVID-19 experience. Curr Treat Options Psychiatry. 2020;7:1-5.

7 Zhou X, Snoswell CL, Harding LE, Bambling $\mathrm{M}$, Edirippulige S, Bai X, et al. The role of telehealth in reducing the mental health burden from COVID-19. Telemed J E Health. 2020; 26(4):377-9.

8 Huremovic D. A mental health response to infection outbreak. Cham: Springer International Publishing; 2019.

9 Shigemura J, Ursano RJ, Morganstein JC, Kurosawa $\mathrm{M}$, Benedek DM. Public responses to the novel 2019 coronavirus (2019-nCoV) in Japan: mental health consequences and target populations. Psychiatry Clin Neurosci. 2020; 74:277-83.

10 Banjar WM, Alaqeel MK. Healthcare worker's mental health dilemma during COVID-19 pandemic: a reflection on the KSA experience. J Taibah Univ Med Sci. 2020; 15(4):255-7.

11 Teoh K, Kinman G. Looking after doctors' mental wellbeing during the COVID-19 pandemic. BMJ Opinion. 2020 [cited 2021 Mar 28]. Available from: https://blogs.bmj.com/bmj/2020/03/ 26/looking-afterdoctors-mental-wellbeing-during-the-covid-19-pandemic.
12 Golberstein E, Wen H, Miller BF. Coronavirus disease 2019 (COVID-19) and mental health for children and adolscents. JAMA Pediatrics. 2020;174(9):819-20.

13 Di Carlo F, Sociali A, Picutti E, Pettorruso M, Vellante F, Verrastro V, et al. Telepsychiatry and other cutting-edge technologies in $\mathrm{CO}$ VID-19 pandemic: bridging the distance in mental health assistance. Int J Clin Pract. 2020;00:1-9.

14 Cowan KE, McKean AJ, Gentry MT, Hilty DM. Barriers to use of telepsychiatry: clinicians as gatekeepers. Mayo CLin Proc. 2019; 94:2510-23.

15 WHO. A health telematics policy in support of WHO's health-for-all strategy for global health development: report of the WHO group consultation on health telematics. 1997.

16 Waugh M, Voyles D, Thomas MR. Telepsychiatry: benefits and costs in a changing health-care environment. Int Rev Psychiatry. 2015;27(6):558-68

17 Caudill R, Wright JH. Remote treatment delivery in response to the COVID-19 pandemic. Psychother Psychosom. 2020.

18 Mohr DC, Ho J, Duffecy J, Reifler D, Sokosl L, et al. Effect of telephone-administered vs faceto-face cognitive behavioral therapy on adherence to therapy and depression outcomes among primary care patients: a randomized trial. JAMA. 2012;307(21):2278-85. 